\title{
Design and realization of magnetic nanoparticles detector based on the nonlinear magnetization
}

\author{
Yang $\mathrm{Yu}^{1, \mathrm{a}}$,Yan Tan ${ }^{1,2, \mathrm{~b}}$, Qian $\mathrm{Li}^{1,2, \mathrm{c}}$, Ming Wang ${ }^{1, \mathrm{~d}^{*}}$ \\ ${ }^{1}$ Institute of electrical engineering Chinese academy of sciences, Beijing, China \\ ${ }^{2}$ University of Chinese Academy of Sciences, Beijing, China

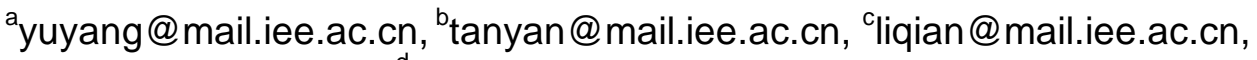 \\ wangming@mail.iee.ac.cn
}

\begin{abstract}
Keywords: nonlinear magnetization, magnetic nanoparticles
Abstract. Magnetic nanoparticles have been widely used in the field of biotechnology and medicine, which has a great importance to precise and quantitative detection. This paper introduces a magnetic nanoparticles detection device of low cost, high sensitivity and real-time. When the diameter of the magnetic nanoparticles is $20 \mathrm{~nm}$, the third harmonic intensity of the induced voltage is in the level of microvolt, and the larger the mass of the magnetic nanoparticles is, the greater the induced signal strength will be. In addition, there is a linear relation between them. The device can distinguish 18ug of magnetic nanoparticles.
\end{abstract}

\section{Introduction}

The application of magnetic nanoparticles in the field of life science started a decade ago[1], of which the special magnetic and binding properties make it widely used in the magnetic detection technology and put forward magnetic detection technology.

The vitro methods of the detection of magnetic nanoparticles are atomic absorption spectrometry [2], electron paramagnetic resonance [3], and inductively coupled plasma mass spectrometry [4]. The methods of quantitative measurement of concentration of the magnetic nanoparticles by measuring the magnetic response in alternating magnetic field are susceptometry and relaxometry. Susceptometry, which lock-in detects the response in a certain frequency oscillating magnetic field, has high sensitivity of quantitative measurement of the concentration of the magnetic nanoparticles but without selectivity, so other paramagnetic substances may influence the measurement result. Relaxometry gets the concentration of magnetic nanoparticles by measuring the magnetic response of the magnetic nanoparticles in a radio frequency pulsed magnetic field and related calculations. Relaxometry can distinguish between bound and unbound magnetic nanoparticles, for bound nanoparticles recovering from pulse magnetic field according to Neel relaxation in milliseconds to seconds while unbound nanoparticles follow Brown relaxation in a microsecond scale. The SQUID, an expensive and complex cooling device, will be used to measure such short time [5].

The newly developed measurement technology based on nonlinear magnetic properties of magnetic nanoparticles can extract the weak effective signal from the background signal, and measure accurately the high order harmonic component by spectrum analysis method, effectively avoiding the disturbance of the base frequency signal of the background signal.

\section{Principles and Materials}

Basic principle. Langevin theory describes the magnetization behaviour of small area of magnetic nanoparticles in thermal equilibrium, which does not appear magnetic hysteresis in external magnetic field. According to the Langevin theory, the magnetization of super-paramagnetic nanoparticles can be expressed as the following approximate Eq. 1 [6]:

$$
M=M_{0} L\left(\frac{H \cdot V \cdot M_{s} \cdot \mu_{0}}{k_{B} \cdot T}\right)
$$


Where the function $L(\alpha)$ is $L(\alpha)=\operatorname{coth}(\alpha)-1 / \alpha, M_{0}$ the saturation magnetization of the sample, $H$ the external magnetic field strength, $V$ the volume of the particle, $M_{s}$ the saturation magnetization of the particle, $k_{B}$ the Boltzmann constant, $T$ the absolute temperature, and $\mu_{0}$ gives the permeability of vacuum. Fig. $1 \mathrm{~b}$ is the magnetization curve of magnetic nanoparticles described by the Langevin theory. Exerted on external sinusoidal excitation magnetic field $H_{e}$ (c.f. Fig. 1a), magnetic nanoparticles will be magnetized and induce a non-sinusoidal magnetic field (c.f. Fig. 1c). Its derivation multiplies a constant related to concentration of the magnetic nanoparticles and pick-up coil is the voltage of the pick-up coil (c.f. Fig. 1d). The spectrum of the voltage signal after Fourier transform is shown in Fig. 1e. Both excitation coil and magnetization of magnetic nanoparticles contribute to the base wave part of the spectrum, and the excitation coil is far greater than that of magnetic particle, so only harmonic signal of testing is taken into account. The exact concentration of the magnetic nanoparticles is thusly measured by the quantitative measurement of Harmonic signal detected by the pick-up coil.

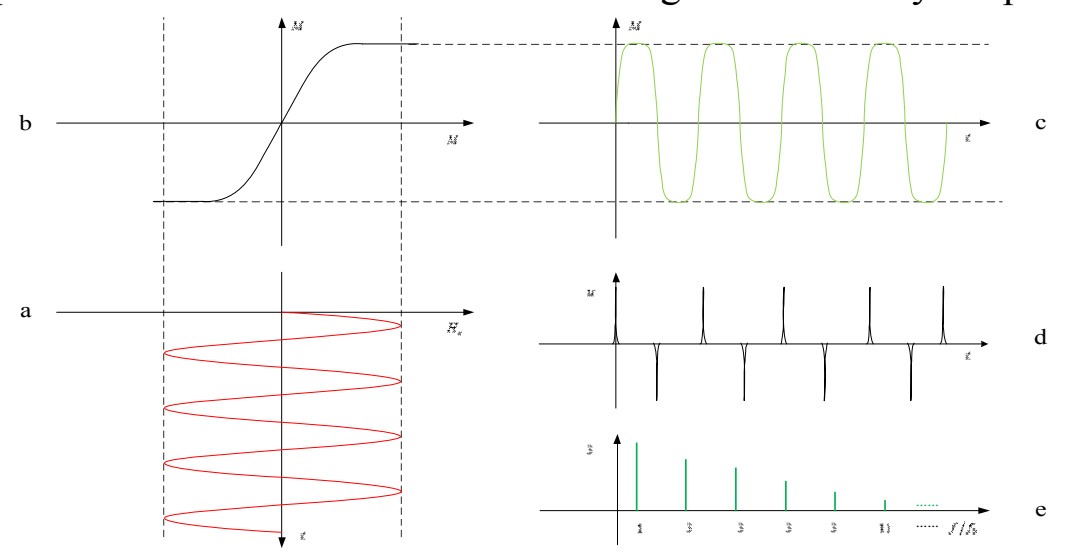

Figure 1. Theory of nonlinear magnetization

Materials. Super-paramagnetic nanoparticle is a special kind of magnetic nanomaterial, which has a super-paramagnetic and good biocompatibility. Super-paramagnetic is a phenomenon that nanoparticle is back to the same state after magnetization and demagnetization. The advantages of super-paramagnetic nanoparticle lead to extensive biotechnological and medical research and application. In area of biotechnology, super paramagnetic nanoparticles are used for separation and purification of proteins and cells separation and purification of DNA/RNA[7], and so on ; in biomedical field, super paramagnetic nanoparticles are used for magnetic resonance imaging, magnetic particle imaging[8], targeted therapy drugs[9], and so on.

\section{Hardware Components}

Magnetic nanoparticle detection apparatus consists of the following components shown in Fig. 2, function signal generator, power amplifier, band-pass filter, coil system, band stop filter, signal amplifier, data acquisition card and PC.

Function signal generator. Function signal generator generates a frequency (such as $25 \mathrm{kHz}$ ) sinusoidal signal. It should ensure the output signal is a sinusoidal signal with constant amplitude and does not contain any harmonic component.

Power amplifier. Since the function generator's output signal is not powerful enough to make excitation coil produce oscillation magnetic field with a certain amplitude, power amplifier is necessary for the detection. Power amplifier output power should be between $30 \mathrm{w}$ and $60 \mathrm{w}$, maximum output current should be between $3 \mathrm{~A}$ and $5 \mathrm{~A}$, and sufficient power amplifier linearity and enough bandwidth are required.

Band-pass filter. Because of performance limit of the electronics, power amplifier output contains different frequency noise. In order to ensure the signal of drive excitation coil is sinusoidal signal with a single frequency, band - pass filter should be connected after power amplifier in series. This can be realized by a four-order Chebyshev filter. 
Coil system. Coil system includes excitation coil and pick-up coil. The excitation coil generates alternating magnetic field with a constant frequency to magnetize the magnetic nanoparticles. The excitation coil can be Helmholtz coil or solenoid. With a certain frequency current, uniform magnetic field can be achieved in a certain area around its axial direction. This article select solenoid as excitation coil. According to Biot - Safar law, magnetic field strength at the centre axis of solenoid by Eq. 2.

$$
H(t)=\frac{N}{2 \sqrt{(l / 2)^{2}+r^{2}}} i(t)
$$

Where $N$ is the number of turns of the coil, $l$ the length of the coil, $r$ the radius of the coil, $i(t)$ the current through the coil. The pick-up coil is wound into a solenoid. Due to the fundamental frequency component of the detection signal (excitation field oscillation frequency) is not useful. To eliminate it, the pick-up coil is designed as a solenoid shown in Figure 3b. Both ends of the solenoid have the same number of turns and winding direction. The sum turns of both ends is equal to that of the intermediate portion and their winding direction are opposite. Coil with these features is known as differential coil.

Band stop filter. To ensure that the output signal from the detection coil contains only harmonics, a band stop filter should be connected to the output of the detection coil in series. Since the main purpose is to prevent the baseband signals, it can be accomplished using high-pass filter. We choose Chebyshev filter here for implementation.

Low noise amplifier. Output effective signal of detection coil output is quite small (millivolt level), detected signal must be amplified in order to match the input signal of data acquisition card.

Signal acquisition and processing. Data acquisition and processing is accomplished by data acquisition card and PC. After Fourier transform of acquired signal and the spectrum can be displayed. The concentration of detected nanoparticles can be obtained after the calibration of the whole detecting system. Thus the ultimate goal can be achieved.

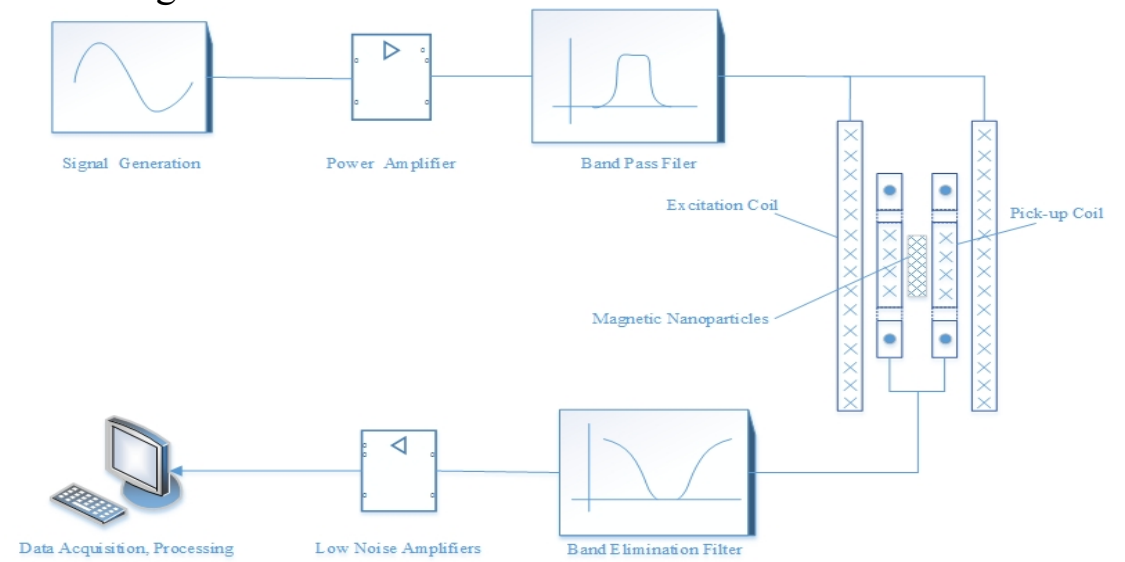

Figure 2. The system schematic of magnetic nanoparticles detection

\section{Results and Discussion}

A detection sample, silica-coated iron oxide nanoparticles with size of $15 \sim 20 \mathrm{~nm}$, was used in this paper. The designed device can detect samples with different mass. The third harmonic component can be achieved after Fourier transform. The results are shown in Fig. 3.

As can be seen from the Fig. 3, the detection device designed in this paper can effectively distinguish magnetic nanoparticles with mass of $18 \mathrm{ug}$, and increasing mass of magnetic nanoparticles made the third harmonic component's intensity stronger. The intensity of the detection signal's third harmonic component had a good linear relationship with magnetic nanoparticles' content. 


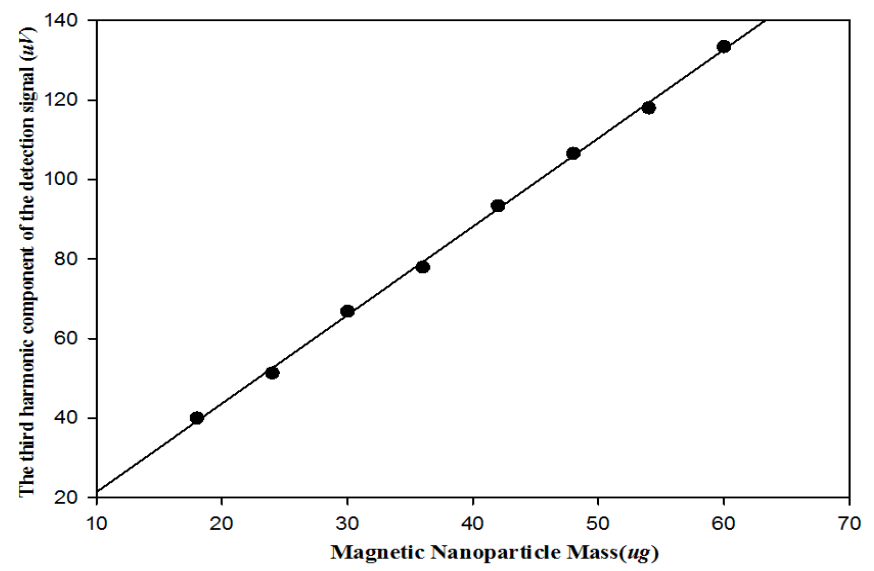

Figure 3. Magnetic nanoparticle mass - signal intensity curve

\section{Conclusions}

In this paper, based on the basic principles and materials, methods based on nonlinear magnetization of the magnetic nanoparticle detection techniques are introduced, presenting, analysing the detection system block diagram and finally verifying the feasibility of detection techniques by experiments.

\section{Acknowledgements}

This study was supported by Grant from the National Natural Science Foundation of China (31300689).

\section{References}

[1] T. M. Buzug, G. Bringout, M. Erbe, K. Grafe, M. Graeser, M. Gruttner, et al., "Magnetic particle imaging: Introduction to imaging and hardware realization," Zeitschrift Fur Medizinische Physik, pp. 323-334, 2012.

[2] M. Settles, M. Etzrodt, K. Kosanke, M. Schiemann, A. Zimmermann, R. Meier, et al., "DifferentCapacity of Monocyte Subsets to Phagocytose Iron-Oxide Nanoparticles," vol. 6 (2011).

[3] L. F. Gamarra, A. J. daCosta, J. B. Mamani, R. D. Ruiz, L. F. Pavon, T. T. Sibov, et al., "Ferromagnetic resonance forthe quantification of superparamagnetic iron oxide nanoparticles in biological materials," International Journal ofNanomedicine, vol. 5, pp. 203-211, 2010.

[4] K. R. Minard, M. H. Littke, W. Wang, Y. Xiong, J. G. Teeguarden, and B. D. Thrall, "Magnetic particle detection(MPD) for in-vitro dosimetry," Biosens Bioelectron, vol. 43, pp. 88-93, 2013.

[5] H. J. Krause, N. Wolters, Y. Zhang, A. Offenhausser, P. Miethe, M. H. F. Meyer, et al., "Magnetic particle detection byfrequency mixing for immunoassay applications," Journal of Magnetism and Magnetic Materials, vol. 311, pp. 436-444,Apr 2007.

[6] J. Borgert, J. D. Schmidt, I. Schmale, J. Rahmer, C. Bontus, B. Gleich, et al., "Fundamentals and applications ofmagnetic particle imaging," Journal of Cardiovascular Computed Tomography, vol. 6, pp. 149-153, May-Jun 2012.

[7] S. Berensmeier, "Magnetic particles for the separation and purification of nucleic acids," Appl Microbiol Biotechnol,vol. 73, pp. 495-504, Dec 2006.

[8] B. Gleich and R. Weizenecker, "Tomographic imaging using the nonlinear response of magnetic particles," Nature,vol.435, pp. 1214-1217, Jun 302005.

[9] L. Brannon-Peppas and J. O. Blanchette, "Nanoparticle and targeted systems for cancer therapy," Advanced DrugDelivery Reviews, vol. 64, pp. 206-212, Dec 2012. 